\title{
Points entiers au voisinage d'une courbe plane de classe $C^{n}$
}

\author{
par
}

\author{
M. N. HuXley (Cardiff) et P. Sargos (Nancy)
}

1. Introduction et énoncé du résultat. Soient $a \in \mathbb{R}, N$ un entier $\geq 2, f:[a, a+N] \rightarrow \mathbb{R}$ une fonction de classe $C^{n}(n \geq 2)$ et $\delta \in \mathbb{R}$ $(0 \leq \delta \leq 1 / 4)$. On pose :

$$
\Gamma_{\delta}=\left\{(x, y) \in \mathbb{R}^{2}|a \leq x \leq a+N,| y-f(x) \mid \leq \delta\right\}
$$

et

$$
\nu\left(\Gamma_{\delta}\right)=\#\left(\Gamma_{\delta} \cap \mathbb{Z}^{2}\right) .
$$

Le problème annoncé dans le titre consiste à majorer $\nu\left(\Gamma_{\delta}\right)$. On fait l'hypothèse :

$$
\left|f^{(n)}(x)\right| \asymp \lambda, \quad \text { pour } a \leq x \leq a+N,
$$

où $\lambda$ est un réel positif "petit". On cherche des majorations de la forme

$$
\nu\left(\Gamma_{\delta}\right) \ll N \lambda^{\alpha_{n}}+N \delta^{\beta_{n}}+\text { termes secondaires, }
$$

où les termes secondaires ne peuvent dominer que dans la situation marginale où $\lambda$ est trop petit.

Les majorations de $\nu\left(\Gamma_{\delta}\right)$ obtenues par des méthodes de sommes d'exponentielles fournissent la valeur optimale $\beta_{n}=1$ mais avec une valeur de $\alpha_{n}$ trop faible. Par exemple, le résultat classique de van der Corput (cf. [3], Théorème 2.8), qui s'applique sous la seule hypothèse (1.2), donne la valeur $\alpha_{n}=1 /\left(2^{n}-1\right)$, avec en outre des termes secondaires élevés.

En se ramenant par un procédé de nature combinatoire au cas facile où $n=1$, le premier auteur [4] a obtenu une amélioration de $\alpha_{n}$, substantielle pour $n$ grand, au détriment de $\beta_{n}$. Le résultat est

(1.4) $\nu\left(\Gamma_{\delta}\right) \ll N \lambda^{2 /(n(n+1))}+N \delta^{2 /(n(n-1)+2)}+\delta \lambda^{-1}(\log N)^{n}+N^{1-1 / n}+1$, sous la seule hypothèse (1.2).

Par une méthode géométrique simple, nous améliorons (1.4) en remplaçant $\beta_{n}=2 /(n(n-1)+2)$ par $\beta_{n}=2 /(n(n-1))$, mais surtout en obtenant les termes secondaires optimaux. Notre résultat s'énonce ainsi. 
THÉORÈme 1. Sous la seule hypothèse (1.2), on a

$$
\nu\left(\Gamma_{\delta}\right) \ll N \lambda^{2 /(n(n+1))}+N \delta^{2 /(n(n-1))}+\left(\delta \lambda^{-1}\right)^{1 / n}+1
$$

(le symbole « sous-entend une constante qui ne dépend que de $n$ et des constantes sous-entendues dans (1.2)).

Dans le cas $n=2$, ce résultat a été obtenu dans [1] par une méthode similaire. Le passage du cas $n=2$ au cas $n \geq 3$ fait apparaître des difficultés dues au fait que les "arcs majeurs" ne sont plus des segments. Par ailleurs, le Lemme 3.4 de [1], sur l'espacement des arcs majeurs, est remplacé par le Lemme 7 qui semble plus adapté et qui permet, dans le cas $n=2$, d'obtenir une importante simplification dans la démonstration de [1].

Concernant les améliorations ultérieures, signalons un article de Filaseta et Trifonov [2] dans lequel l'exposant $\beta_{n}$ est meilleur que le nôtre dès que la dérivée $(n-1)$-ième est suffisamment petite, et que certaines contraintes sont satisfaites.

Cependant, à cause de la simplicité de nos hypothèses, et surtout à cause des termes secondaires, l'intérêt de notre résultat subsiste.

Notations. Soient $u$ et $v$ deux réels positifs. La notation $: u \ll v$ signifie: $u \leq A v$, où $A$ est une constante qui ne dépend que de $n$ et des constantes introduites antérieurement. Comme d'habitude, $u \asymp v$ signifie qu'on a à la fois : $u \ll v$ et $v \ll u$.

La lettre $M$ désigne systématiquement un élément de $\mathbb{Z}^{2}$ (point entier ou point à coordonnées entières), d'abscisse $m$ et d'ordonnée $l: M=(m, l)$, $M_{i}=\left(m_{i}, l_{i}\right), \ldots$

Si $E$ est une partie du plan, $\nu(E)$ est le nombre de points entiers de $E: \nu(E)=\#\left(E \cap \mathbb{Z}^{2}\right)$. Si $\mathcal{P}$ est une famille de parties du plan, on pose

$$
\nu(\mathcal{P})=\nu\left(\bigcup_{E \in \mathcal{P}} E\right) .
$$

Dans tout ce qui suit, on suppose $n \geq 2$.

2. Espacement des points isolés. Nous présentons deux lemmes de base. Le premier a été introduit dans le problème par Swinnerton-Dyer [5] et généralise, d'une certaine façon, la formule des accroissements finis. Le deuxième, qui est une conséquence du premier, montre que si $n+1$ points entiers de $\Gamma_{\delta}$ sont "en position générale", alors ils sont bien espacés. Cela permet déjà de montrer que le nombre de points sur la courbe $(\operatorname{cas} \delta=0)$ vérifie

$$
\nu\left(\Gamma_{0}\right) \ll N \lambda^{2 /(n(n+1))}+1 .
$$


Lemme 1. Soit $g:[a, b] \rightarrow \mathbb{R}$ une fonction $C^{k}$. Etant donnés $a \leq x_{0}<$ $x_{1}<\ldots<x_{k} \leq b$, on désigne par $Q(x)$ l'unique polynôme de degré $\leq k$ vérifiant $Q\left(x_{i}\right)=g\left(x_{i}\right)$ pour $i=0,1, \ldots, k$. Alors il existe $\left.\xi \in\right] x_{0}, x_{k}[$ tel que $Q^{(k)}(\xi)=g^{(k)}(\xi)$.

La démonstration consiste en une application répétée du Théorème de Rolle (cf. [5]). Rappelons que la formule d'interpolation de Lagrange qui donne $Q(x)$ s'écrit :

$$
Q(x)=\sum_{j=0}^{k}\left(\prod_{i \neq j} \frac{x-x_{i}}{x_{j}-x_{i}}\right) g\left(x_{j}\right),
$$

où la notation abrégée $\prod_{i \neq j}$ signifie $\prod_{0<i<k, i \neq j}$.

Pour le lemme suivant, nous n'aurons pas besoin de l'hypothèse (1.2), mais seulement de la propriété :

$$
\left|f^{(n)}(x)\right| \leq \mu \quad \text { pour } a \leq x \leq a+N .
$$

Lemme 2. Soient $M_{0}, M_{1}, \ldots, M_{n} n+1$ points entiers de $\Gamma_{\delta}$, vérifiant $a \leq m_{0}<m_{1}<\ldots<m_{n} \leq a+N$, et qui ne sont pas tous sur une même courbe polynômiale de degré $<n$. Alors, sous l'hypothèse (2.2), on a

$$
m_{n}-m_{0} \geq \min \left(\mu^{-2 /(n(n+1))}, \frac{1}{6} \delta^{-2 /(n(n-1))}\right) .
$$

Par courbe polynômiale de degré $<n$, on entend une courbe $y=P(x)$ où $P$ est un polynôme de degré $<n$, éventuellement nul.

Démonstration. Posons $f\left(m_{i}\right)=l_{i}+\delta_{i},\left|\delta_{i}\right| \leq \delta, i=0, \ldots, n$. Par le Lemme 1, on peut écrire

$$
\frac{1}{n !} f^{(n)}(\xi)=\sum_{j=0}^{n} \frac{l_{j}+\delta_{j}}{\prod_{i \neq j}\left(m_{j}-m_{i}\right)} .
$$

De même, si $P(x)=\sum_{j=0}^{n} b_{j} x^{j}$ est le polynôme d'interpolation des $M_{i}$ $(0 \leq i \leq n)$, alors on a

$$
b_{n}=\sum_{j=0}^{n} \frac{l_{j}}{\prod_{i \neq j}\left(m_{j}-m_{i}\right)}=\frac{u}{v},
$$

avec $u \in \mathbb{Z}$ et $v=\prod_{0 \leq i<j \leq n}\left(m_{j}-m_{i}\right)$. Par hypothèse, $b_{n} \neq 0$, donc $|u| \geq 1$, d'où

et donc

$$
\frac{1}{v} \leq \frac{1}{n !}\left|f^{(n)}(\xi)\right|+\sum_{j=0}^{n}\left|\frac{\delta_{j}}{\prod_{i \neq j}\left(m_{j}-m_{i}\right)}\right|
$$

$$
1 \leq \frac{1}{n !} \mu\left(m_{n}-m_{0}\right)^{n(n+1) / 2}+(n+1) \delta\left(m_{n}-m_{0}\right)^{n(n-1) / 2},
$$

et (2.3) en découle. 
3. Propriétés des arcs majeurs. Les points entiers de $\Gamma_{\delta}$ qui se regroupent sur une même courbe polynômiale de degré $<n$ forment des arcs majeurs, et leur contribution, plus difficile à évaluer, fait l'objet du reste de cet article. On commence par quelques propriétés générales.

Dans ce paragraphe, on remplace l'hypothèse (1.2) par la suivante :

$$
0<\lambda \leq\left|f^{(n)}(x)\right| \quad \text { pour } a \leq x \leq a+N .
$$

Soit $P(x)=\sum_{j=0}^{n-1} b_{j} x^{j}$ un polynôme à coefficients rationnels; on désigne par $q$ le plus petit entier $\geq 1$ tel que $P \in \frac{1}{q} \mathbb{Z}[X]$, et par $\gamma$ la courbe

$$
\{(x, y) \mid x \in \mathbb{R}, y=P(x)\} .
$$

DÉfinition. Un arc majeur, d'équation $y=P(x)$, est une composante connexe $\mathcal{A}$ de $\gamma \cap \Gamma_{\delta}$ vérifiant $\nu(\mathcal{A}) \geq n+1$. Le dénominateur de $\mathcal{A}$ est l'entier q. La longueur de $\mathcal{A}$ est la longueur de la projection de $\mathcal{A}$ sur l'axe des $x$.

Lemme 3. Au plus $n$ arcs majeurs ont la même équation $y=P(x)$.

Démonstration. On prolonge, pour simplifier, $f$ en une fonction $\widetilde{f}$ : $\mathbb{R} \rightarrow \mathbb{R}$ de classe $C^{n}$ vérifiant $\widetilde{f}^{(n)}(x)=f^{(n)}(a)$ si $x<a$ et $\widetilde{f}^{(n)}(x)=$ $f^{(n)}(a+N)$ si $x>a+N$, de sorte que $\left|f^{(n)}(x)\right| \geq \lambda$ pour $x \in \mathbb{R}$.

Comme les comportements à l'infini de $\tilde{f}$ et de $P$ sont différents, $\gamma \cap \widetilde{\Gamma}_{\delta}$ est borné, avec

$$
\widetilde{\Gamma}_{\delta}=\{(x, y)|x \in \mathbb{R},| \widetilde{f}(x)-y \mid \leq \delta\},
$$

si bien que chaque composante connexe $\mathcal{A}$ de $\gamma \cap \widetilde{\Gamma}_{\delta}$, non réduite à un point, possède deux extrémités vérifiant $P(x)=f(x) \pm \delta$. Supposons qu'il existe $n+1$ arcs majeurs; alors il existerait $n+1$ composantes connexes de $\gamma \cap \widetilde{\Gamma}_{\delta}$, non réduites à un point, et l'équation $P(x)=f(x)+\delta$ (par exemple) admettrait $n+1$ solutions $x_{0}, x_{1}, \ldots, x_{n}$. Par le Lemme 1 , on aurait

$$
0=\frac{P^{(n)}(\xi)}{n !}=\sum_{j=0}^{n} \frac{P\left(x_{j}\right)}{\prod_{i \neq j}\left(x_{j}-x_{i}\right)}=\sum_{j=0}^{n} \frac{P\left(x_{j}\right)-\delta}{\prod_{i \neq j}\left(x_{j}-x_{i}\right)}=\frac{f^{(n)}\left(\xi_{1}\right)}{n !} \neq 0,
$$

avec contradiction.

Lemme 4. La longueur d'un arc majeur est $\leq 2 n(\delta / \lambda)^{1 / n}$.

Démonstration. Soient $y=P(x)$ l'équation d'un arc majeur $\mathcal{A}$ et $L$ la longueur de $\mathcal{A}$. Ecrivons $\varphi(x)=f(x)-P(x)$ et $\mathcal{A}=\{(x, y) \mid c \leq x \leq$ $c+L, y=P(x)\}$.

On pose alors $x_{j}=c+j L / n$ pour $0 \leq j \leq n$. Le Lemme 1 montre alors que

$$
\frac{f^{(n)}(\xi)}{n !}=\frac{\varphi^{(n)}(\xi)}{n !}=\sum_{j=0}^{n} \frac{\varphi\left(x_{j}\right)}{\prod_{i \neq j}\left(x_{j}-x_{i}\right)}
$$


avec $\left|\varphi\left(x_{j}\right)\right| \leq \delta$ et $\left|f^{(n)}(\xi)\right| \geq \lambda$, si bien que

$$
\frac{\lambda}{n !} \leq \delta\left(\frac{n}{L}\right)^{n}\left(\frac{1}{n !}+\frac{1}{(n-1) ! 1 !}+\ldots+\frac{1}{n !}\right)=\delta\left(\frac{n}{L}\right)^{n} \frac{2^{n}}{n !},
$$

d'où le résultat.

Lemme 5. Un arc majeur $\mathcal{A}$, de longueur $L$ et de dénominateur q vérifie

$$
\nu(\mathcal{A}) \leq(n-1)\left(L q^{-2 /(n(n-1))}+1\right) .
$$

Démonstration. Considérons $n$ points $M_{1}, \ldots, M_{n} \in \mathcal{A}$ avec $m_{1}<$ $m_{2}<\ldots<m_{n}$. Si $y=P(x)$ est l'équation de $\mathcal{A}$, alors

$$
P(x)=\sum_{j=1}^{n} l_{j} \prod_{i \neq j} \frac{x-m_{i}}{m_{j}-m_{i}} .
$$

En particulier, $q$ divise $\prod_{1 \leq i<j \leq n}\left(m_{j}-m_{i}\right) \leq\left(m_{n}-m_{1}\right)^{n(n-1) / 2}$, d'où $m_{n}-m_{1} \geq q^{-2 /(n(n-1))}$, d'où le lemme.

4. Espacement des arcs majeurs. Nous montrons dans ce paragraphe que les arcs majeurs dont le dénominateur n'est pas trop grand, sont bien espacés. Nous commençons par une propriété intermédiaire générale :

Lemme 6. Soit $\varphi:[c, c+L] \rightarrow \mathbb{R}$ une fonction $C^{n}$ vérifiant

$$
|\varphi(x)| \leq \delta \quad \text { et } \quad\left|\varphi^{(n)}(x)\right| \leq \mu \quad \text { pour } c \leq x \leq c+L .
$$

Alors pour tout $x \in[c, c+L]$ et chaque $k=1,2, \ldots, n-1$, on a

$$
\left|\varphi^{(k)}(x)\right| \leq\left(2^{n} n^{n-1} \delta+\mu L^{n}\right) L^{-k} .
$$

Dém onstration. Etant donné $k(1 \leq k \leq n-1)$, on écrit le polynôme d'interpolation de $\varphi$ aux points $c, c+L / k, c+2 L / k, \ldots, c+L$. En raisonnant comme au Lemme 4 , on obtient

$$
\left|\frac{\varphi^{(k)}\left(\xi_{k}\right)}{k !}\right| \leq \frac{(2 k)^{k}}{k !} \cdot \frac{\delta}{L^{k}}, \quad \text { pour un certain } \xi_{k}\left(c<\xi_{k}<c+L\right) .
$$

On utilise la formule de la moyenne :

$$
\left|\varphi^{(k)}(x)\right| \leq\left|\varphi^{(k)}\left(\xi_{k}\right)\right|+L \sup _{t}\left|\varphi^{(k+1)}(t)\right|
$$

pour majorer successivement $\sup \left|\varphi^{(n-1)}(x)\right|, \sup \left|\varphi^{(n-2)}(x)\right|, \ldots, \sup \left|\varphi^{\prime}(x)\right|$, et on obtient (4.2).

Pour le lemme suivant, nous n'aurons besoin que de l'hypothèse (2.2).

Lemme 7. Soit $\mathcal{A}$ un arc majeur, d'équation $y=P(x)$, de longueur $L$ et de dénominateur $q$, avec $q \leq 1 /(3 \delta)$. Soient $M_{0} \in \mathcal{A}$ et $M \in \Gamma_{\delta}$. On suppose 
que $M$ n'est pas sur la courbe $y=P(x)$. Alors

$$
\left|m_{0}-m\right| \gg \min \left(L(q \delta)^{-1 /(n-1)},(q \mu)^{-1 / n}, \frac{1}{q \mu L^{n-1}}\right) .
$$

Démonstration. Posons $m=m_{0}+h$ et supposons, par exemple, $h>0$. Soit $\varphi(x)=f(x)-P(x)$ et soit $[c, c+L]$ la projection de $\mathcal{A}$ sur l'axe des $x$. On a

$$
\varphi(m)-\varphi\left(m_{0}\right)=\sum_{k=1}^{n-1} \varphi^{(k)}\left(m_{0}\right) \frac{h^{k}}{k !}+\varphi^{(n)}(\xi) \frac{h^{n}}{n !}
$$

pour un certain $\xi \in[c, c+L]$.

Mais $\left|\varphi(m)-\varphi\left(m_{0}\right)\right| \gg q^{-1}$. En effet, comme $M=(m, l)$ vérifie $P(m) \neq$ $l$, on a $|P(m)-l| \geq 1 / q \geq 1 /(3 q)+2 \delta$; par suite

$$
\left|\varphi(m)-\varphi\left(m_{0}\right)\right| \geq|\varphi(m)|-\delta \geq|P(m)-l|-|f(m)-l|-\delta \geq 1 /(3 q) .
$$

Maintenant, on majore $\left|\varphi^{(k)}\left(m_{0}\right)\right|$ par le Lemme 6 et on reporte ces inégalités dans (4.4) :

$$
q^{-1} \ll\left(\delta+\mu L^{n}\right) \sum_{k=1}^{n-1}\left(\frac{h}{L}\right)^{k}+\mu h^{n},
$$

et (4.3) en découle.

Voici maintenant la formulation particulière du Lemme 7 dont nous aurons besoin.

Lemme 8. On suppose que $\left|f^{(n)}(x)\right| \asymp \lambda$ pour $x \in[a, a+N]$. Alors $i l$ existe une constante $B$ qui ne dépend que de $n$ et des constantes sousentendues dans (1.2) telle que, sous la condition: $q \leq 1 /(B \delta)$, et sous les hypothèses de Lemme 7 , on ait:

$$
\left|m_{0}-m\right|>L
$$

et

$$
\left|m_{0}-m\right| \gg \min \left(L(q \delta)^{-1 /(n-1)},(q \lambda)^{-1 / n}\right) .
$$

La démonstration est une conséquence immédiate de (4.3) et du Lemme 4.

5. Démonstration du Théorème. Dans tout ce paragraphe, on suppose que $f$ vérifie (1.2). Nous allons démontrer un résultat légèrement plus précis que le Théorème 1 :

ThÉORÈme 2. Soit $\mathcal{P}$ l'ensemble des arcs majeurs de $\Gamma_{\delta}$. Alors on a

$$
\nu\left(\Gamma_{\delta}\right) \ll N \lambda^{2 /(n(n+1))}+N \delta^{2 /(n(n-1))}+\max _{\mathcal{A} \in \mathcal{P}} \nu(\mathcal{A})+1 .
$$


Le Théorème 2 implique le Théorème 1 puisque $\nu(\mathcal{A}) \ll\left(\delta \lambda^{-1}\right)^{1 / n}$, d'après le Lemme 4.

Dém o n s tration. Soit $\mathcal{P}_{0}$ l'ensemble des arcs majeurs dont le dénominateur est $\leq 1 /(B \delta), B$ étant la constante du Lemme 8 , et soit $\mathcal{R}$ l'ensemble des points entiers de $\Gamma_{\delta}$ qui ne proviennent pas de $\mathcal{P}_{0}$. On majore séparément $\nu\left(\mathcal{P}_{0}\right)$ et $\nu(\mathcal{R})$.

(a) Majoration de $\nu(\mathcal{R})$. On considère $n^{2}+1$ points $M_{0}, M_{1}, \ldots, M_{n^{2}}$ de $\mathcal{R}$ (avec $m_{0}<m_{1}<\ldots<m_{n^{2}}$ ). Si ces points ne sont pas tous sur une même courbe polynômiale de degré $<n$, alors le Lemme 2 montre que

$$
m_{n^{2}}-m_{0} \gg \min \left(\lambda^{-2 /(n(n+1))}, \delta^{-2 /(n(n-1))}\right) \text {. }
$$

S'ils sont sur une courbe polynômiale de degré $<n$, avec dénominateur $q>1 /(B \delta)$, alors le Lemme 5 montre que : $m_{n^{2}}-m_{0} \gg q^{2 /(n(n-1))}$, et (5.2) est encore vérifié.

Il n'y a pas de troisième alternative, car si les points étaient tous sur une courbe polynômiale de degré $<n$, avec dénominateur $q \leq 1 /(B \delta)$, alors, d'après le Lemme $3, n+1$ d'entre eux seraient sur un arc majeur $\mathcal{A} \in \mathcal{P}_{0}$, ce qui est impossible. Donc (5.2) est toujours vérifié, d'où

$$
\nu(\mathcal{R}) \ll N \lambda^{2 /(n(n+1))}+N \delta^{2 /(n(n-1))}+1 .
$$

(b) Majoration de $\nu\left(\mathcal{P}_{0}\right)$. Quitte à multiplier la majoration obtenue par $n$, on peut supposer, d'après le Lemme 3 , que deux arcs majeurs de $\mathcal{P}_{0}$ n'ont jamais la même équation.

On désigne par $\sigma$ la projection $\mathbb{R}^{2} \ni(x, y) \rightarrow x \in \mathbb{R}$. D'après (4.5), les $\sigma(\mathcal{A})$ ne se chevauchent pas; on peut donc, pour chaque $\mathcal{A} \in \mathcal{P}_{0}$, associer le plus grand intervalle de $[a, a+N]$, noté $I(\mathcal{A})$, contenant $\sigma(\mathcal{A})$, mais ne rencontrant pas $\sigma\left(\mathcal{A}_{1}\right)$ pour tout $\mathcal{A}_{1} \neq \mathcal{A}$. Si $|I|$ représente la longueur d'un intervalle $I$, on a

$$
\sum_{\mathcal{A} \in \mathcal{P}_{0}}|I(\mathcal{A})| \leq 2 N
$$

Par ailleurs, si $\mathcal{A}$ est de longueur $L$ et de dénominateur $q$, on a, d'après (4.6),

$$
|I(\mathcal{A})| \gg \min \left(L(q \delta)^{-1 /(n-1)},(q \lambda)^{-1 / n}\right),
$$

sauf peut-être si $\mathcal{A}$ est à l'une des extrémités de $[a, a+N]$. D'autre part, par le Lemme 5 , on a

$$
\begin{aligned}
\nu(\mathcal{A}) & \ll L q^{-2 /(n(n-1))} \ll|I(\mathcal{A})|\left(\frac{(q \delta)^{1 /(n-1)}}{L}+(q \lambda)^{1 / n}\right) L q^{-2 /(n(n-1))} \\
& \ll|I(\mathcal{A})| \delta^{2 /(n(n-1))},
\end{aligned}
$$

la dernière inégalité prenant en compte le fait que $q \ll \delta^{-1}$ et que $L \ll$ $(\delta / \lambda)^{1 / n}$. On reporte cette inégalité dans (5.4), en prenant soin d'ajouter la 
contribution des arcs majeurs situés aux extrémités, ce qui donne :

$$
\nu\left(\mathcal{P}_{0}\right) \ll \max _{\mathcal{A} \in \mathcal{P}_{0}} \nu(\mathcal{A})+N \delta^{2 /(n(n-1))} .
$$

Le Théorème 2 est entièrement démontré.

\section{Bibliographie}

[1] M. Branton et P. Sargos, Points entiers au voisinage d'une courbe plane à très faible courbure, Bull. Sci. Math. 118 (1994), 15-28.

[2] M. Filaset a and O. Trifonov, The distribution of fractional parts with applications to gap results in Number Theory, à paraître.

[3] S. W. Graham and G. Kolesnik, Van der Corput's Method of Exponential Sums, London Math. Soc. Lecture Note Ser. 126, Cambridge University Press, 1991.

[4] M. N. Huxley, The integer points close to a curve, Mathematika 36 (1989), 198-215.

[5] H. P. F. Swinnerton-Dyer, The number of lattice points on a convex curve, J. Number Theory 6 (1974), 128-135.

SCHOOL OF MATHEMATICS

UNIVERSITY OF WALES COLLEGE OF CARDIFF

INSTITUT ELIE CARTAN

23, SENGHENYDD ROAD

UNIVERSITÉ DE NANCY I

CARDIFF, CF2 4YH, WALES, U.K.

B.P. 239

54506 VANDOEUVRE CEDEX, FRANCE

Reçu le 25.11.1992

et révisé le 14.10.1994 